\title{
3D VISUALIZATION FOR VIRTUAL MUSEUM DEVELOPMENT
}

\author{
M. Skamantzari ${ }^{\text {a }}$, A. Georgopoulos ${ }^{\text {a }}$

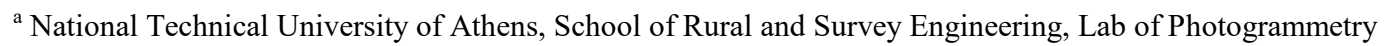 \\ Zografou Campus, Heroon Polytechniou 9, 15780, Zografou, Athens, Greece \\ sk.margarita@hotmail.com,drag@central.ntua.gr
}

Special Session SpS1, CIPA

KEY WORDS: Virtual Museum, 3D models, 3D survey

\begin{abstract}
:
The interest in the development of virtual museums is nowadays rising rapidly. During the last decades there have been numerous efforts concerning the 3D digitization of cultural heritage and the development of virtual museums, digital libraries and serious games. The realistic result has always been the main concern and a real challenge when it comes to 3D modelling of monuments, artifacts and especially sculptures. This paper implements, investigates and evaluates the results of the photogrammetric methods and 3D surveys that were used for the development of a virtual museum. Moreover, the decisions, the actions, the methodology and the main elements that this kind of application should include and take into consideration are described and analysed. It is believed that the outcomes of this application will be useful to researchers who are planning to develop and further improve the attempts made on virtual museums and mass production of 3D models.
\end{abstract}

\section{INTRODUCTION}

The 3D virtual spaces and modern technology are more and more used especially in the development of applications in elearning and edutainment by cultural organisations and museums. The abilities, the restrictions and the limits of technology in this area have not yet been explored and defined, as the advantages that virtual reality offers constantly increase. The virtual museum is a sub-category of $3 \mathrm{D}$ virtual spaces and it is usually a replica of a real museum (Moldoveanu et al., 2008). There is not a generally accepted definition and many different terms are used to designate it. However, the virtual museum can be defined "... as a logically related collection of digital objects composed in a variety of media which, because of its capacity to provide connectedness and various points of access, lends itself to transcending traditional methods of communicating and interacting with visitors...; it has no real place or space, its objects and the related information can be disseminated all over the world" (Schweibenz, 2004).

\section{SCOPE AND MEANS OF VIRTUAL MUSEUMS}

Today there are four different categories of museum available online that are created and developed as an extension to the real museum: the brochure museum, the content museum, the learning museum and the virtual museum (Schweibenz, 2004). The virtual museum is considered to include all the benefits of all the other categories and at the same time it is an innovative and alternative product. It provides the visitors with all the necessary information about the museum and its collections and at the same time it connects all the available digital collections from every museum around the world. A simple search on the term "virtual museum" on the web gives almost 17.400 .000 results. However, the majority of the results have nothing to do with the 3D virtual spaces where the user can take a virtual tour and explore digital collections and 3D models in an interactive way. So it is important to understand what exactly a virtual museum should include and what are the benefits and the advantages of these applications both for the museum and the visitors.

\subsection{Main Elements in a 3D Virtual Museum}

The creation and development of a virtual museum is a rather challenging task with many different aspects that should be taken into consideration. The most important are the requirements' analysis, the architectural design, the planning of the exhibits' presentation, the user interaction, the programming process and the evaluation of the final product (Lepouras et al., 2004).

The first task includes the recording of the museums' visitors and demands in order to form a detailed profile. The museum characteristics such as the aim of the museum, its needs, exhibitions and collections should be identified and recorded as they are vital to the development of the virtual museum and its content. The process of the architectural design aims to support and adjust the visitor into the virtual environment during the navigation in the museum in order to maintain a sense of orientation. The overall museum structure expands in three dimensions and there have been developed different types of structure with both advantages and disadvantages (Lepouras et al., 2004). Moreover, as far as the level of realism is concerned, the task of design offers two alternatives, either to depict the museum in the most realistic way possible or to design a novel museum based on different and alternative 3D models, not necessarily of a realistic form. In both cases all the elements of the virtual environment and the result of the museum need to have a complete, clear and compact structure.

The museum, virtual or real, communicates specific messages to its visitors through exhibiting its content, so the task of design also includes the design of the exhibit presentation. The virtual museum gives the curators the opportunity to experiment and try alternative routes through the content of new exhibitions before they make their final choices and the most important is that they have the chance to duplicate exhibits in a virtual 
museum or represent them in a different place and time, something that they cannot do in the real world museum.

Furthermore, many interactive elements can enrich the virtual environment of a museum, in order not to be static and with limited possibilities. Generally, there are different techniques for navigation and interaction but the most common and proper is the technique that enables the visitor to navigate or teleport in the virtual museum and allows him/her to manipulate an exhibit when he/she gets close to it.

The programming task and evaluation of the final project are based on the decisions and requirements of both the visitors and the museum. Except from the 3D models of the building, the exhibits and the primary objects of the virtual environment, other elements need to be programmed. The architectural objects are usually 3D meshes of high complexity and can be obtained by various means, i.e. existing plans and models, survey measurements, photogrammetric methods or laser scanning. Usually in order to develop a more realistic, accurate, appealing and efficient virtual museum, all the above methods are combined and used along with proper algorithms and software for 3D reconstruction of the 3D models. Moreover, the realistic result of the models requires high resolution textures, which are images mapped to the 3D surfaces by different methods.

Finally all the decisions and changes that were made during the previous tasks of analysis, design and evaluation need to be taken into consideration in every step and task of the process and the virtual museum is developed based on them. The final product is an intuitive, user-friendly, complete and rigid virtual museum ready to open its doors for the public.

\subsection{The Advantages of Virtual Museums}

The development of a virtual museum does not only offer a more vivid, realistic and appealing experience to the visitors, but the advantages that these applications have are more important to the museum itself and to the curators. First of all, the lack of space is one of the main reasons why the virtual museums should be considered, as most of the museums display only a small part of their collection (Lepouras et. al., 2004). Moreover, some objects can be extremely fragile or valuable to be exhibited, but not in a virtual environment especially when the visitor is able to interact with them and learn all the essential information about them. Another reason that makes virtual museums more valuable is the possibility they offer to the visitors to view a virtual reconstruction of important objects, buildings, or environments that may no longer exist, or are damaged.

The digitisation of cultural heritage is important as it helps to preserve it and make it accessible for a wider audience in a more appealing and contemporary way. The technological progress and the wide use of internet and social media can make the digitised content of a museum more accessible to all. It can be easily added and transported to any kind of mobile application in order to address to a wider audience and exhibition sites from all around the world. As a result, the virtual museums acts in a complementary and auxiliary manner, making its content and its exhibitions more interesting, exhilarating and appealing to the visitors. The website of a museum and the applications of virtual reality can provide worldwide publicity to important monuments, archaeological places and exhibits. The result of the Colorado's University research is quite notable, according to which $70 \%$ of the people who visit a museum website would subsequently be more likely to go and also visit the real museum (Loomis R.J. et al., 2003). Moreover, the museum curators can digitally preserve the artifacts from the permanent or temporary exhibitions (Sylaiou et al., 2009). The pertinent information and data can be stored, renewed and retrieved whenever needed, for every purpose. Virtual museums provide access from any place and to anyone, including people with special needs, like visual, acoustic, speech and motor disabilities or learning difficulties.

In virtual museum exhibitions, the visitor is able to fully control his/her navigation in the museum as well as to freely explore, move around, manipulate the exhibits and create his/her own virtual experience in an interactive and flexible way. It is also very important that the visitor can create, save and share a personal collection of 3D digital exhibits even from different museums according to his/her interests and preferences. Moreover, in a virtual museum the visitor is not just an observer but he/she interacts with the exhibits via a constructive dialogue and enriches his/her knowledge. The overall process and navigation of the visitor in the virtual museum enhances the understanding, awakens and keeps the interest alive by enriching the aesthetic sensitivities. Finally, the museums and the curators can benefit by making changes, plans and experiments with the 3D models of the exhibits and by finding the best position for them both in the virtual and the real museum.

\section{THE VIRTUAL MUSEUMS OF TODAY}

Today the development of virtual museums and online libraries are intensive. The efforts and the organisations that work on the development of virtual museums in Europe and all over the world are numerous. A country could benefit from the cultural sector both socially and economically (EURO INNOVANET, 2008). It is worth mentioning that the contribution of the cultural sector to the European Economy is extremely important and the different advantages and goods that come from it should be highlighted in both economic and social terms.

\subsection{Projects and Efforts on Cultural Heritage}

The projects related to cultural heritage that took place in Europe mostly started in 2000 and the majority of them were funded by the European Union. The first efforts were made by the MU.S.EU.M. Project, MINERVA Project and the Michael Project (EURO INNOVANET, 2008). The aim of these attempts was to help public museums join the digital and virtual world of today, by training the staff and creating an efficient and profitable virtual museum in Europe according to the standards. In Greece, the two main organisations concerning the production, development and digitisation of cultural heritage was at first the Society of Information (www.ktpae.gr) from 2000 until 2006 and then the Digital Convergence (www.digitalplan.gov.gr) that replaced and continued the actions and work of the Society of Information. The initiatives of these organisations include many projects that concern the cultural sector and some of the most recent projects are "The virtual guide to Byzantine Architecture: Aghia Sophia a World Heritage Site" and "The cultural navigator of Athens in GPS systems and mobile phones".

During the time that these projects were active and running, in 2006 a survey was conducted by the Universities of Pittsburgh and North Carolina about the museum uses and users (Griffiths 
and King, 2007). In particular the survey mostly focused on online users who remotely visit the museums, but also addressed to traditional museum visitors. According to the survey and as it is presented in Table $1,70 \%$ of 223 million adults visited museums in-person or remotely through the Internet in the past 12 months.

\begin{tabular}{|c|c|c|c|c|}
\hline \multirow{2}{*}{} & \multicolumn{2}{|c|}{ In-person visit } & \multicolumn{2}{c|}{ Remote visit } \\
\cline { 2 - 5 } & Visitors & Visits & Visitors & Visits \\
\hline Total male & 66.04 & 335.05 & 36.38 & 263.77 \\
\hline Total female & 70.11 & 310.54 & 35.65 & 219.33 \\
\hline Unknown & 11.66 & 55.33 & 6.17 & 40.65 \\
\hline ALL & $\mathbf{1 4 7 . 8 1}$ & $\mathbf{7 0 0 . 9 2}$ & $\mathbf{7 8 . 2 0}$ & $\mathbf{5 2 3 . 7 5}$ \\
\hline
\end{tabular}

Table 1: Number of visits and visitors to museums by adults, inperson and remotely in 2006.

It is very important to observe and highlight the fact that the traditional visits to the museum are more when the user has the chance to remotely visit the museum through the internet. It is clear and confirmed from the survey that virtual museums and online collections and libraries have a great impact and contribute to the increase of visitors in the real museum.

The year 2008 could be considered a milestone as the initiatives, the projects and ideas concerning the cultural heritage, its digitisation and the development of museums, were numerous and many of the programmes are running until today. For example Europeana is an online library that hosts 43 million exhibits and the organisations that contribute to it are more than 2.500 (www.psvaluetalks.gr). The digitisation of cultural heritage plays a determinant role in the preservation, promotion and development of cultural heritage and even in the economic growth of the countries. Only $10 \%$ of cultural heritage has been digitised until today and it includes different aspects of the European culture, as it is captured in books, paintings, photographs, letters, sounds, music and videos (www.statistics.europeana.eu). In 2010 another project began in Europe, the Learning Museum (LEM) and in 2014 this project was integrated in the Network of European Museum Organisations (NEMO). Their goal was to create a wide network which will offer to every museum and academic a permanent place to meet, exchange ideas, knowledge and methods in order to contribute to the creation of a European community that will be interested in cultural heritage and museums (Sani, 2013). NEMO today has over 30.000 members across Europe (www.ne-mo.org) and its mission is to ensure that museums are an integral part of European life by promoting their work and value to policy makers and by providing museums with information, networking and opportunities for co-operation.

Another European Union project was the Linked Heritage project which started in 2011 (www.linkedheritage.org). The main goal of this project was to contribute large quantities of new content to Europeana, from both public and private sectors, but also to demonstrate enhancement of quality content, in terms of metadata richness, re-use potential and uniqueness. The project achieved its goals with 3 million entries and digital information in Europeana's library which were linked to data and information from other sources.

Finally in 2011 Google developed an online platform, the Google Art Project, where the public has access to high quality images of artifacts from all over the world (www.psvaluetalks.gr). The collection has today about
6.000.000 exhibits from 60 countries and the users can take a virtual tour inside 17 of the world's most acclaimed art museums with Street View technology and create and share online their own digital collections (www.googleblog.blogspot. gr). In addition, every museum has the chance to select one artwork that is photographed in extraordinary detail using super high resolution photo capturing technology, the so called gigapixel image.

The majority of online and virtual museums struggles to find balance between spatial and cognitive information. Some great examples that achieved to make the museums venues for social change and educational tools are The Tech Virtual by Second Life (www.thetechvirtual.org), the Valentino Garavani Virtual Museum (http://www.valentinogaravanimuseum.com) and the Acropolis Museum (www.theacropolismuseum.gr). Second Life is a $3 \mathrm{D}$ virtual world in which users can move through spaces, teleport in museums, interact with objects, find information and communicate with other users (www.interactive.org). The users have the chance to virtually build and display their own collection and among them there are many professional initiatives for developing and promoting cultural institutions. There are mainly two kinds of museums: the virtual versions of real-life museums and museums completely designed and conceived for Second Life (Fig. 2). Moreover, SL offers historical reconstructions such as the Sistine Chapel or Ancient Rome.

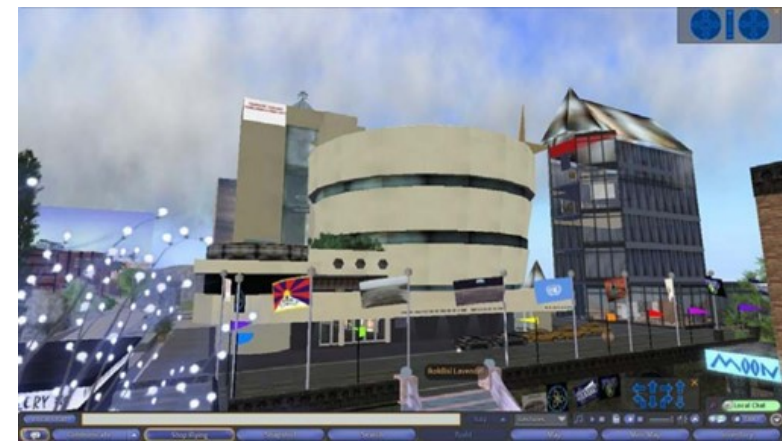

Figure 2: The Newggenheim Museum of Second Life.

The Valentino Garavani Virtual Museum is a virtual 3D museum that does not really exist. This effort gathers all the main elements of a successful virtual museum and it is open to everyone online. The museum was developed in the platform of Unity $3 \mathrm{D}$ and gives the chance to the visitor to freely navigate in the museum, explore and interact with the exhibits and find useful information about them. Finally, the Acropolis Museum hosts in its website a variety of online applications, educational programmes and serious games. All these applications are quite interactive and especially the virtual visit "Athena, the Goddess of Acropolis" (Fig. 3) which gives the chance to the visitor to navigate in the museum, teleport to points of interest, interact with the exhibits, learn about them and manipulate them.

\subsection{Technological Means and Systems in Cultural Sector}

Museums offer and provide their visitors with surroundings for touring them in order to achieve knowledge. Countries all over the world are using museums as a core, central facility to promote culture and tourism by widening the collections, exhibits and services (Tsai et al., 2010). Thanks to recent advances in information and networking technology, along with the increase in ownership of wireless network devices, the majority of museums started to construct wireless guidance 
systems and adopted technological means and systems so as to offer a more appealing, interesting, educational and complete experience to each visitor individually.

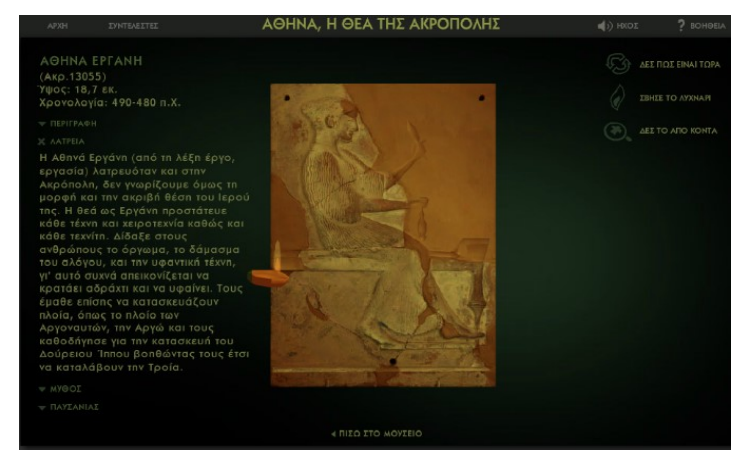

Figure 3: Exhibit from the online application "Athena, the Goddess of Acropolis"

One of the most common systems used in a museum are the digital guide systems that are able to enrich the visitor's experience and give him/her more valuable information. The visitors are more likely to remember information from a recorded guided tour rather than by reading boards (Schwarzer, 2001). Nowadays, the technological means and systems that are mostly used in cultural sector are:

- The audio tour guides. (Wallace, 2006 and Razaq et al., 2013).

- The location based services (LBS), using global navigation satellite systems (GNSS), unique markers like barcodes, image matching techniques, infrared or Bluetooth beacons, radio frequency identification tags (RFID), WiFi positioning, radio triangulation or mobile phone triangulation methods. (Hornecker et al., 2006).

- Quick Response (QR) codes (Winter, 2011).

- Holography (www.hih.org.gr).

- 3D technology and digital products from survey and photogrammetric methods

- 3D printing (Digitech III, 2005 and Prokos, 2012).

\section{THE VIRTUAL MUSEUM OF THE STOA OF ATTALOS}

This paper focuses on the development of a virtual museum that hosts some of the exhibits which can be found on the ground floor of the Stoa of Attalos, in the Ancient Agora of Athens. The Stoa of Attalos, on the east side of the Ancient Agora, was a gift of Attalos II, King of Pergamon and was built between $159-138$ B.C. The two floor building was $120 \mathrm{~m}$ long and $20 \mathrm{~m}$ wide and it was destroyed by the Heruli in 267 A.D. and it was fully reconstructed in 1953-1956. Now, it is the museum of the Ancient Agora of Athens and all of the finds from the excavations are kept and displayed there. For this project, only 16 of the exhibits were chosen from the south part of the open colonnade of the museum. The development of the virtual museum was confined to this part, in order to investigate the implementation of the technological tools and the impact of their outcomes.

\subsection{Data Acquisition}

Simple instrumentation was employed in order to obtain the necessary data for the development of the virtual museum, since (a) there was no need to reference the exhibits to a specific system and (b) only correct scale should be attributed to the 3D models of the exhibit. The data acquired was mainly digital images, as it was decided to implement image based techniques for producing the $3 \mathrm{D}$ models. The following equipment was used:

- A Nikon D3200 DSLR camera with a CMOS sensor $23.2 \times 15.4 \mathrm{~mm}$, pixel size of $5 \mu \mathrm{m}$ and resolution of $24 \mathrm{Mpixel}$

- A Nikkor AF-S DX 18-55mm zoom lens with f/3.5-5.6 aperture values respectively.

- A special photographic tripod

- A collapsible light reflector, in order to direct lighting to the dark areas of the exhibits

- A $1 \mathrm{~m}$ long steel ruler with 4 pre-marked targets precisely measured, in order to give scale to the models

- A ladder (!)

The purpose of the image acquisition process was to take suitable sets of digital images in order to create the 3D models of the 16 exhibits using Structure from Motion and Multi View Stereo techniques.

\begin{tabular}{|c|c|c|c|c|c|}
\hline Item & Quality & $\begin{array}{c}\text { Dense } \\
\text { Cloud (\# } \\
\text { of points) }\end{array}$ & $\begin{array}{c}3 D \\
\text { Model } \\
\text { Faces }\end{array}$ & $\begin{array}{c}\text { File } \\
\text { Size } \\
\text { (MB) }\end{array}$ & $\begin{array}{c}\# \text { of } \\
\text { Imgs }\end{array}$ \\
\hline$\# 1$ & Low & 1.455 .587 & 28.447 & 38 & 178 \\
\hline$\# 2$ & Low & 988.864 & 16.367 & 27 & 71 \\
\hline$\# 3$ & Medium & 3.673 .587 & 222.998 & 51 & 65 \\
\hline$\# 4$ & Medium & 3.820 .015 & 193.318 & 50 & 54 \\
\hline$\# 5$ & Low & 584.202 & 13.533 & 24 & 41 \\
\hline$\# 6$ & Low & 1.477 .870 & 28.310 & 37 & 155 \\
\hline$\# 7$ & Low & 709.948 & 16.405 & 30 & 88 \\
\hline$\# 8$ & Low & 574.407 & 17.090 & 26 & 33 \\
\hline$\# 9$ & Low & 2.050 .721 & 38.581 & 40 & 101 \\
\hline$\# 10$ & Low & 1.475 .243 & 28.272 & 37 & 86 \\
\hline$\# 11$ & Medium & 5.061 .722 & 313.568 & 62 & 66 \\
\hline$\# 12$ & Medium & 1.604 .071 & 107.386 & 33 & 39 \\
\hline$\# 13$ & Low & 429.369 & 16.369 & 22 & 42 \\
\hline$\# 14$ & Low & 3.704 .639 & 218.984 & 54 & 88 \\
\hline$\# 15$ & Medium & 1.391 .898 & 27.305 & 31 & 60 \\
\hline$\# 16$ & Medium & 1.446 .480 & 69.466 & 31 & 41 \\
\hline
\end{tabular}

Table 4: The quantitative details of the 3D models

The amount of images varied for each item, according to its size and complexity (Table 4). In total 1208 images were taken for all the exhibits, most of them using the tripod for more stability, during three visits and the whole process lasted approximately 11 hours. The average amount of images for each exhibit was $60-90$, while some of them needed more (100-120) and some less (30-45) images according to their size and complexity. While the images were acquired, the scale bar, i.e. the ruler, was placed next to each exhibit so as to appear in at least 3 images for scaling the exhibit later in the process of the 3D modelling.

\subsection{Data Processing and 3D Modelling}

PhotoScan Professional v.1.1 software by Agisoft was used to process the images and build the 3D models of the exhibits 
(www.agisoft.com). It is an image based modelling (IBM) software, meaning that it automatically generates $3 \mathrm{D}$ models based on images. It is a piece of software that gives the chance to the user to interfere or not, at certain points during the overall procedure of the 3D modelling in order to set the parameters for the process. The interference of the user is sometimes very critical and requires deep photogrammetric knowledge.

The images for each exhibit were loaded in separate projects. The images were examined for their quality, as blurred or badly lit images obstruct the automated algorithm and lead to erroneous results. Then, the 4 pre-marked targets of the scale bar were detected on each image and they were marked, while their world coordinates were introduced to the software in order to assign scale for each $3 \mathrm{D}$ model.

The next steps of the software are:

- The image orientation, where the algorithm searches for common points on the images, matches them and finds the position of each image refining the parameters of the interior camera geometry. The matching procedure is based on a variation of the Scale Invariant Feature Transformation (SIFT) algorithm. The parameters that could be adjusted by the user at this stage were (i) the image quality, (ii) the way the software manages the image pairs and (iii) the number of point to be determined for the orientation.

- The generation of the dense point cloud, using dense image matching techniques based on the estimated image positions. The dense clouds were later edited using another software in order to delete the unnecessary points and noise. The parameters that could be adjusted by the user at this stage concern the accuracy of the point determination and the quality of the depth calculation.

- The generation of the 3D polygonal mesh which represents the exhibit's surface based on the dense point cloud (Figure 5). The mesh was reconstructed using the Arbitrary option recommended for complicated surfaces.

- The texturing of the mesh, after the reconstruction of the geometry using the Generic mapping mode to take care of the complicated surfaces of the exhibits.

The precision was decided not be high in this kind of applications, as it concerns the proof of concept. Hence, the parameters on every step and stage of the 3D modelling were chosen to be of low or medium quality, as it appears in Table 4. In that way the $3 \mathrm{D}$ models built could be easily imported in the game engine environment later for the development of the virtual museum. The fast, easy and mass production of realistic and high quality $3 \mathrm{D}$ models is an important aspect for this kind of applications.

The models were exported in .obj format (Wavefront OBJ) and their texture in .tiff format. During the export it was important to also export the vertex normals, the directional vectors associated with a vertex as a replacement to the true geometric normal of the surface, in the geometry of computer graphics.

\subsection{Evaluation of the Virtual Museum Software}

There are numerous pieces of software allowing the handling, editing and presenting of 3D models. Especially only for the development of virtual museums, some of the available software are VEX-CMS (www.hcilab.uniud.it/vex/) and 3DSSE, while some platforms like ArcGIS and Unity can also be used for that purpose and also have interesting features and possibilities.
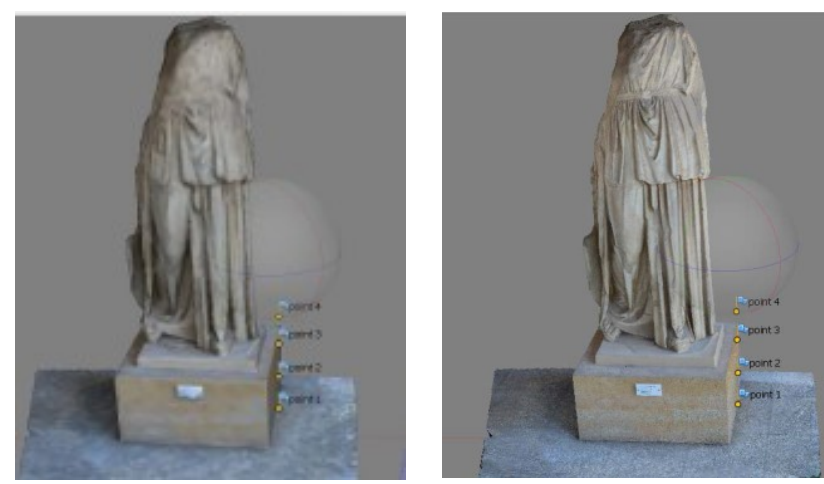

Figure 5: The 3D polygonal mesh on the left and the textured 3D mesh on the right

VEX-CMS is an application that allows users with no expertise in $3 \mathrm{D}$ modelling to create $3 \mathrm{D}$ virtual exhibitions and virtual visits integrating objects and information into virtual environments using existing 3D models of the virtual spaces and of the objects and artworks that are part of the exhibition. (Chittaro L. et al., 2010). It can be used by museum curators and employees without expertise on 3D models and virtual reality, but it gives them the opportunity to find proper points of interest about the exhibits and the museum in general and also to experiment with the position of the exhibits before an exhibition is organised.

3DSSE is a 3D scene search engine that facilitates virtual visits in complex 3D scenes by employing a keyword-based search engine (Koutsoudis A. et al., 2012). The idea of this engine is to annotate the 3D scenes with information that enclose archaeological details, historical facts and spatial relationships of entities, which the visitor can identify as points of interest and explore them by using keyword-based queries. A great improvement to this application is a X3DOM-WebGL compatible version that eliminates the need of installing a VRML/X3D plug-in on the user's Web browser so as to handle more complex queries. X3DOM is a new, easy and innovative way to present $3 \mathrm{D}$ scenes and models online at websites that replace VRML and plug-ins.

In addition, the platform of ArcGIS by Esri with the proper Extensions (Spatial, 3D Analyst, Server and Online) and the environments of ArcScene and ArcGlobe proved to be inappropriate for virtual applications and navigations. Firstly, the platform does not have many interactive possibilities and the user cannot freely navigate in the environment or manipulate and explore the exhibits and objects. Moreover, the developer of the application loses the copyrights of the 3D models and environment the moment it is imported in the environment of ArcGIS. This platform is more appropriate for animations and videos in environments with 3D models, online applications and interactive maps, etc.

\subsection{The Unity 3D Platform}

Unity is a cross-platform game engine developed by Unity Technologies (www.docs.unity3d.com) and is used to develop video games and virtual applications for PC, consoles, mobile devices and websites. This platform was preferred for the development of the virtual museum since it was considered as 
the most suitable and appropriate. This engine offers a plug-in which is compatible to all known browsers. Unity is also able to export the applications and games that are developed as independent applications for mobile devices. The most important advantage of Unity is that it gives the opportunity to the user to write and develop his own scripts or use the ones that it offers. The game engine's scripting is built on Mono, the open-source implementation of the .NET Framework. Programmers can use UnityScript which is based on JavaScript, $\mathrm{C} \#$ or Boo that is based on Python. The scripts that are available can be used for the programming process, the design of graphic effects, the control and behaviour of the avatar and the elements in general of the virtual environment.

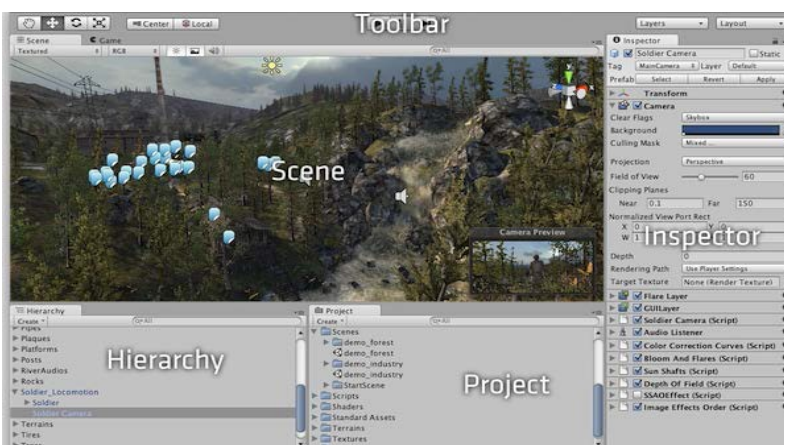

Figure 6: The main Interface and Views of Unity

When starting any project in Unity, the user needs to familiarise himself with the interface (Fig. 6) and the main editor window that is made up of several tanned panels known as Views and each one has specific purpose. The Scene View is the interactive sandbox where the user selects, positions, manoeuvres and manipulates environments, the visitor, the camera and all the other Game Objects. Then the Game View is rendered from the Camera(s) in the environment and it is representative of the final product and application. In the Project Browser the user can access and manage the assets that belong to the project and the left panel contains the folder structure of the project as hierarchical list, with its contents. The Hierarchy contains every Game Object in the current Scene. The most common components that are used in the Game Objects and 3D models that are imported are the mesh with its texture, shader and way of lighting and effects. These elements are essential and crucial for the virtual environment and the result of the application. Finally, the Console window shows errors, warnings and other messages generated by Unity to aid with debugging.

\subsection{The Stoa of Attalos in Unity}

The development of a virtual museum within Unity is a challenging and complex process, which demands the familiarisation of the user with the environment and the tools that are available. The first stage of the development of the virtual museum was the organisation of the Assets. In particular the folders were:

- Audio. It included a sound file that was used as a sound effect during the virtual tour.

- Materials. It included the files that represented the shader and material of each exhibit's texture.

- Scripts. It included all the scripts that were written or used in $\mathrm{CH}$.

- Static Meshes. It included the .obj files of the 16 exhibits as they were exported from the software of PhotoScan.
- Textures. It included the tiff files that described the exhibits' texture.

- Standard Assets. It included all the files that Unity provides to the user in order to describe, design and set the Game Objects and Components of the virtual environment.

- Editor. It included the scripts that Unity has so as to apply for the graphic effects.

For every .obj and .tiff file that was imported, a corresponding Material was added in order to describe every $3 \mathrm{D}$ model. It is important to mention that the Shader of each Material was chosen to be Unlit/Texture, so as every 3D model to appear in the virtual environment exactly as it was exported from PhotoScan. Then, all the 3D models were added in the Hierarchy View in order to appear in the Scene View and they were set as children under a common parent. Every exhibit had a higher entity in the hierarchy, an empty Game Object that was placed to the centre of the 3D model and then both of them as one object was placed to the appropriate position in the virtual environment of the museum, as it is in real life. This was an important step for the manipulation and rotation of the exhibits.

The next step was the creation of the visitor's avatar as a Game Object (Fig. 7). This entity included the sound effect and a Camera with the appropriate components and properties in order to control and set the parameters that concern the movement, vision and behaviour of the visitor. In order to optimise the visitor, a capsule was added from the assets of Unity, while the virtual tour was set as a first-person view. The script that was used to control the visitor's movement was available in Unity and the parameters that were set concerned the speed of movement, the rotation and the height of the visitor.

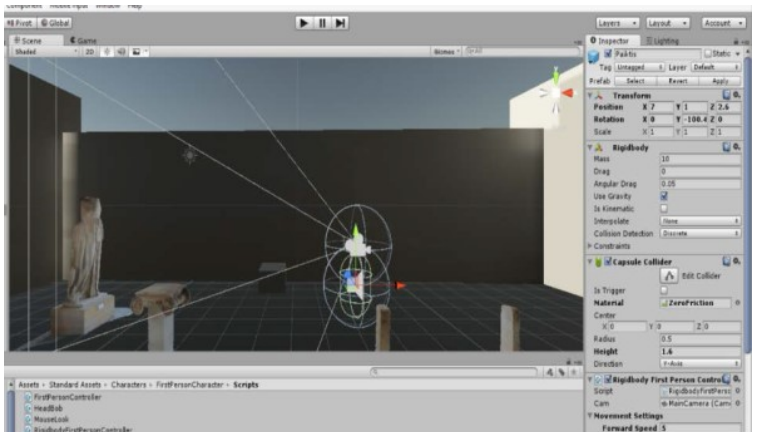

Figure 7: The main elements that configure the entity of visitor in the environment of Unity

As far as the Main Camera is concerned, the components that were added, adjusted the ambience and the depth of field in order to have a more clear and realistic view of the exhibits. The entity of the Directional Light was used to set the lighting conditions of the virtual environment. Furthermore, the proper scripts were added as components to every exhibit in order to achieve the manipulation and rotation of them around the central axis as well as the emergence of information when the visitor selects each one. A script was written for each exhibit according to which whenever the visitor chooses it, he/she is able to rotate it by pressing $\mathrm{Q}$ or $\mathrm{E}$ and at the same time a panel appears on the right part of the screen with all the information about it. A script called "SystemSettings" was also written which allows the visitor to exit the virtual museum by pressing the Esc key. Particular attention was given to the formation and design of the panel that displays the instructions of the virtual 
museum at the beginning of the virtual experience in order to guide and help the visitor move around and explore the environment. In the Hierarchy tab a series of Game Objects from the UI (User Interfaces) tools were imported such as a panel, a framework, a scrollbar, a sliding area, a text box and an exit button. All these elements were edited and positioned properly to form a dialog box where the instructions and the description of each exhibit are shown.

Finally, the last element that was added in the virtual environment was a mini-map (Fig. 8) to help the visitor move and orientate in the environment. A secondary camera was added to follow the visitor and offer a view from the top.

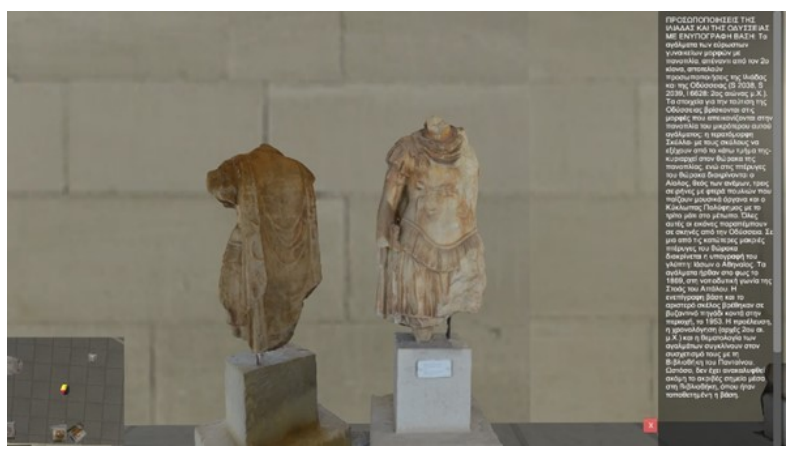

Figure 8: A snapshot from the virtual museum. The visitor chose to rotate the left sculpture, while the available information appears on the right of the screen. The mini-map can be seen on the bottom left of the screen

In a few words, the Virtual Museum of the Stoa of Attalos gives from the beginning the chance to the visitor to read the instructions that are available in order to freely navigate in the environment and understand the options and opportunities he/she has in the virtual museum (Fig. 9). During the navigation the visitor listens to the music that is used as a sound effect so as to have a more appealing, pleasant, entertaining and interesting navigation. Then, the visitor has the chance to learn and find out more information about the exhibits that attract and interest him/her simply by clicking at them. A panel with the information appears on the right part of the screen and he/she is able to interact with the exhibit, manipulate, examine closely and observe the details of it. The visitor is free to exit the virtual museum by pressing the Esc key whenever he/she chooses to do so. Finally, the application is available in two languages, Greek and English.

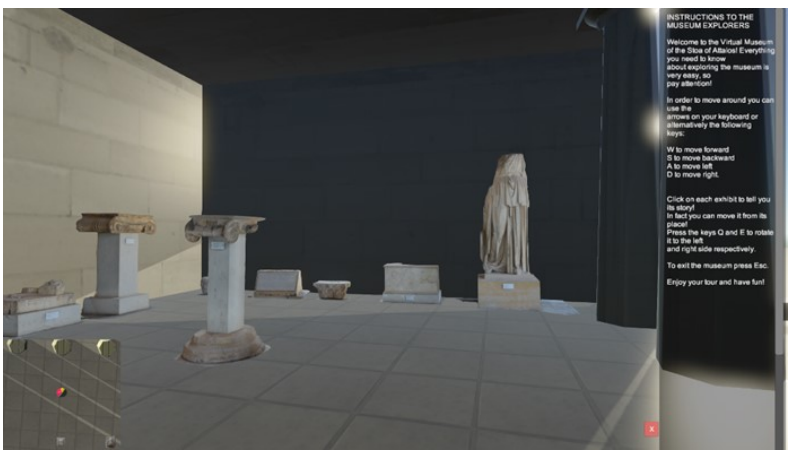

Figure 9: At the beginning of the virtual tour the visitor reads the available instructions

The integration of all the above elements was a complex and complicated procedure that required constant testing and control at every step of the development of the virtual environment. The result is a visitor friendly environment, easy to use and understand, while it maintains the accuracy of the 3D models and information and offers many interactive possibilities.

\section{EVALUATION AND CONCLUDING REMARKS}

The objective of this paper was to highlight the use and exploitation of 3D models in general, that can result from a photogrammetric process and be further used to alternative and innovative products like virtual museums, virtual reconstructions, serious games, etc. The production of $3 \mathrm{D}$ models with photogrammetric methods is a procedure that can definitely give precise and realistic results.

The 3D modelling and presentation of monuments is a rather challenging and complicated process, which requires detail and precision. The acquisition of data, which was the first step in the overall procedure, is a rather demanding and timeconsuming process. There were certain factors that should be taken into consideration like the lighting conditions in the site, the access to every exhibit from all the aspects, which was limited as they were placed next to the walls and it was difficult to always obtain proper images. Moreover, the complexity, the size and details of the exhibits made even more demanding the process of collection of data in the Stoa of Attalos. It is easy to deal with all these issues and overcome the obstacles by taking more images and of course with proper organisation and full recognition and understanding of the site and the exhibits. However, these issues are also reflected on the second step of the development of the virtual museum, which is the production of the 3D models. A lack of information was observed in the part of the exhibits that was extremely close to the walls, especially on their bases, and as a result it was difficult to build their mesh and render their surface properly. Moreover, the problems caused by lighting conditions could be easily solved by processing and improving the images in Photoshop. The process of production of $3 \mathrm{D}$ models in PhotoScan did not present particular difficulties and problems, except for the ones that are mentioned above and concern the acquisition of data.

The last step in the overall procedure was the programming process and the development of the virtual museum in the platform of Unity. This was the most challenging and difficult part, as it is a new and unfamiliar process to the survey engineers. Unity is a highly complex platform, with a variety of functions, features and tools that make it hard and timeconsuming to familiarise with it and gain a good knowledge of all its features. Unity is mainly used by programmers and game designers, who of course have better knowledge of it and could definitely improve and programme a virtual museum in a more appealing, effective, interactive and friendly way to the visitor. Although, the result and the virtual museum that was developed is a really good example, with most of the main elements that it should include and it is quite interesting, appealing and easy to use. Some of the improvements that could be made concern the manipulation of the objects, which could be programmed in a different scene view in Unity, one for each exhibit, separately from the rest of the virtual tour. In that way the visitor would be able to rotate the exhibit, zoom in and out and focus on the exhibit and related information in more detail, without getting distracted. The development, programming and connection of multiple scenes in Unity are complicated processes that require a different level of expertise in this field. In addition, more graphic effects would have a better visual result and would give a much more vivid, realistic and impressive feeling to the 
visitor. Graphic effects that concern the lighting conditions, the improvement of the mini-map, the avatar, the environment around the museum, the orientation of the visitor and perhaps a real-time change of the sun's position could highly improve the visitor's experience and the virtual environment as a whole. Moreover, it would be extremely useful and practical to add points of interest and external links and multimedia in the virtual environment for both the exhibits and the museum. Another possible improvement is to give to the visitor the chance to teleport in the virtual environment, by using hot spots. Finally, some supplementary elements would be useful and educational for the visitors such as predefined tours for different groups of virtual visitors (children, academics, experts, etc.) and serious games or applications at the end of the tour, so as to check what they learned during their virtual experience.

All these issues are food for thought and further research, concern and study. The final and most significant concluding remark of this paper is that with the cooperation of various professionals, such as survey engineers, architects, computer programmers, archaeologists, museum curators, and many others, great and innovative ideas and products can be implemented with significant results. The development of a virtual museum requires an interdisciplinary approach and this is the element that makes it special and impressive to the public.

\section{REFERENCES}

\subsection{References from books:}

Cultural and Educational Technology Institute, Research and Innovation Center in Information, Communication and Knowledge Technologies, 2005. Digitech III: Study of threedimensional digitization technologies. 338pp.

EURO INNOVANET, 2008. Beyond The Traditional Museum. Character, profile and extent of European Virtual Museums. FMU.S.EU.M. Project, Italy, Rome, 151pp.

Razaq R., Griffin K., Morpeth N.D., 2013. Cultual Tourism, CABI Publishing, ISBN 978-18-45939-23-6, 260pp.

Wallace M.A., 2006. Museum branding: how to create and maintain image, loyalty, and support, AltaMira Press, ISBN 978-0-7591-0992-6, 199pp.

Winter M., 2011. Scan Me. Everybody's Guide to the Magical World of QR Codes. Barcodes, Mobile Devices and Hyperlinking the Real to the Virtual, Westsong Publishing, ISBN 978-0-9659000-3-4, 135pp.

\subsection{References from other literature:}

Chittaro L., Ieronutti L., Ranon R., Visintini D., Siotto E., 2010. A High-Level Tool for Curators of 3D Virtual Visits and its Application to a Virtual Exhibition of Renaissance Frescoes. Proceedings of VAST 2010: 11th International Symposium on Virtual Reality, Archaeology and Cultural Heritage, Eurographics/Blackwell Publishing, Oxford, UK, September 2010, pp. 147-154.

Hornecker E., Bartie P., 2006. Technology in Tourism: Handheld Guide Systems and Museum Technologies, Human Interface Technology Laboratory New Zealand, Technical Report TR-2006-1, 28pp.
Griffiths, J-M., King D.W., 2007. Physical Spaces and Virtual Visitors: The Methodologies of Comprehensive Study of Users and Uses of Museums. In: International Cultural Heritage Informatics Meeting (ICHIM07): Proceedings, J. Trant and D. Bearman (eds). Toronto: Archives \& Museum Informatics. Published October 24, 2007 at http://www.archimuse.com/ ichim07/papers/griffiths/griffiths.html

Koutsoudis A., Arnaoutoglou F., Pavlidis G., 2015. Practical Implementation of a web based virtual museum. Using the $\mathrm{X} 3 \mathrm{DOM}$ framework to deliver 3D content on the Web, Proceedings of DIGARCH 2015 NOTES, 19pp.

Lepouras G., Katifori A., Vassilakis C., Haritos D., 2004. Real Exhibitions in a Virtual Museum. In: Journal of Virtual Reality, Vol.7, Issue 2, pp. 120-128.

Loomis R.J., Elias S.M., Wells M., 2003. Website availability and visitor motivation: An evaluation study for the Colorado Digitization Project. Unpublished Report. Fort Collins, CO: Colorado State University, available at: http://www.cdpheritage.org/resource/reports/loomis_report.pdf

Moldoveanu A., Moldoveanu F., Soceanu A., Asavei V., 2003. A 3D Virtual Museum, U.P.B. Sci. Bull., Series C, Vol.70, No.3, ISSN 1454-234x.

Prokos A., 2012. Development of a photogrammetric laser scanner, using further additional geometric constraints. Diploma Thesis. National Technical University of Athens, School of Rural and Survey Engineering, Athens, 171pp. (in Greek).

Sani M., Nicholls A., Pereira M., 2013. Report 7: New trends in museums of the 21st century. The Learning Museum Network Project, Istituto dei Beni Culturali, Italy, ISBN 978-88-9728113-9, 141pp.

Schwarzer M., 2001. Art \& Gadgetry - The Future of the Museum Visit. In: Museum News, 80:4 (July-August 2001).

Schweibenz W., 2004. Virtual Museums. The Development of Virtual Museums. In: ICOM News, Vol.57, No.3.

Sylaiou S., Liarokapis F., Kotsakis K., Patias P., 2009. Virtual museums, a survey and some issues for consideration. In: Journal of Cultural Heritage, Elsevier, 10, ISSN: 1296-2074, pp. 520-528.

Tsai Chih-Yung, Chou Shuo-Yan, Lin Shih-Wei, 2010. Location-aware tour guide systems in museums. In: Scientific Research and Essays Vol. 5(8), pp. 714-720, 18 April. Available online at http://www.academicjournals.org/SRE

\subsection{References from the website}

www.agisoft.com (last visited in June 2015)

www.digitalplan.gov.gr (last visited in August 2015)

www.docs.unity3d.com (last visited in August 2015)

www.googleblog.blogspot.gr/2011/02/explore-museums-and-

www.hcilab.uniud.it/vex/ (last visited in May 2015)

www.hih.org.gr/ (last visited on August 2015)

www.interartive.org/2009/11/virtual-museums/ (last visited in July 2015)

www.ktpae.gr (last visited in August 2015)

www.linkedheritage.org (last visited in June 2015)

www.minervaeurope.org (last visited in June 2015)

www.psvaluetalks.gr (last visited on May 2015)

www.theacropolismuseum.gr (last visited on August 2015)

www.thetechvirtual.org (last visited on July 2015)

www.valentinogaravanimuseum.com (last visited on June 2015) 